\title{
IgG3 subclass: A possible trigger of mixed cryoglobulin cascade in hepatitis $C$ virus chronic infection
}

\author{
Umberto Basile $^{a}$, Francesca Gulli ${ }^{\mathrm{b}}$, Laura Gragnani ${ }^{\mathrm{c}, *}$, Elisa Fognani ${ }^{\mathrm{c}}$, Cecilia Napodano ${ }^{\mathrm{a}}$, \\ Krizia Pocino ${ }^{a}$, Anna Linda Zignego ${ }^{c}$, Gian Ludovico Rapaccini ${ }^{d}$ \\ a Department of Laboratory Medicine, Catholic University of the Sacred Heart, Largo A. Gemelli, 8, 00168 Rome, Italy \\ b Department of Laboratory Medicine, Madre Giuseppina Vannini Hospital, via Acqua Bullicante, 4, 00177 Rome, Italy \\ c Center for Systemic Manifestations of Hepatitis Viruses (MaSVE), Department of Experimental and Clinical Medicine, Department of Experimental and \\ Clinical Medicine, University of Florence, Largo Brambilla, 3, 50134 Florence, Italy \\ ${ }^{\mathrm{d}}$ Institute of Internal Medicine, Catholic University of the Sacred Heart, Largo A. Gemelli, 00168 Rome, Italy
}

\section{A R T I C L E I N F O}

\section{Article history:}

Received 10 February 2017

Received in revised form 3 June 2017

Accepted 6 June 2017

Available online $\mathrm{xxx}$

Keywords:

IgG subclasses

Mixed cryoglobulinemia

Rheumatoid Factor

\begin{abstract}
A B S T R A C T
HCV is a hepatotropic and lymphotropic virus and is the most frequent cause of "benign" mono-oligoclonal B-lymphocyte proliferation, observed in mixed cryoglobulinemia (MC). The study aims to investigate the presence, prevalence and characteristics of the subclasses of cryoglobulins in HCV-patients to look for a relationship with MC. Fifty HCV-infected patients with cryoglobulins were enrolled. IgG subclasses were characterized in cryoprecipitate, and serum IgG and IgM Rheumatoid Factor (RF) were determined.

Patients were stratified into two subgroups according to the presence of IgG3 subclass. Differences were observed in supernatant IgM, IgG3-positive and IgG3-negative patients with a higher IgM concentration in the IgG3-negative cohort $(p=0.03)$. Higher IgM-RF was detected in the $\operatorname{IgG3}$-negative group $(p=0.01)$. IgG3-positive group showed higher IgG-RF compared to the IgG3-negative group $(p<0.0001)$.

IgG3-negative/monoclonal-IgM patients had higher cryocrit compared to IgG3-negative/polyclonalIgM patients $(p<0.01)$. C4 levels were higher in the polyclonal-IgM group compared to monoclonal-IgM group $(p<0.01)$.

We speculate that cryoglobulins are part of a progressive clonal selection process in which, B-cells are stimulated to produce oligoclonal IgG3 with RF activity.

The persistence of the antigenic stimulus elicits the production of polyclonal IgM-RF and subsequently the formation of oligoclonal IgG/polyclonal IgM containing cryoglobulins. In the last stage, a monoclonal IgM-RF clone is formed which may coexist with a monoclonal IgG3-RF clone.
\end{abstract}

(c) 2017 Editrice Gastroenterologica Italiana S.r.l. Published by Elsevier Ltd. All rights reserved.

\section{Introduction}

HCV chronic infection, is often asymptomatic for many years, and can lead to progressive liver damage [1]. The virus is able to infect different sets of blood cells, mainly B lymphocytes [2], which is one of the most likely origins of "benign" monooligoclonal B lymphocyte proliferation, and is typically observed in mixed cryoglobulinemia (MC) [3]. The production of immune complexes, including cryoglobulins (CGs) and various autoantibodies is determined by the activation of B-lymphocytes [4]. CGs

\footnotetext{
* Corresponding author at: Department of Experimental and Clinical Medicine, University of Florence, Largo Brambilla, 3, 50134 Firenze, Italy. Fax: +39055 2758099 .

E-mail addresses: laura.gragnani@unifi.it, lauragragnani@gmail.com (L. Gragnani).
}

are immune-complexes which undergo reversible precipitation below $37{ }^{\circ} \mathrm{C}$ and re-dissolve upon re-warming [5]. Therefore, the mechanism of cryoprecipitation may be secondary to the intrinsic chemical physical properties of the immunoglobulins or to the interaction of individual components of the cryoprecipitate [6]. In types II and III MC, the CGs are composed of polyclonal IgG (behaving as auto-antigens) and IgM, with Rheumatoid Factor (RF) activity, monoclonal in type II, and polyclonal in type III [7].

MC is the most frequent and strongly associated extrahepatic manifestation of HCV: as reported in different studies, more than $90 \%$ of patients with MC are HCV-positive $[2,8]$. MC is both an autoimmune and B-lymphoproliferative disorder; it is clinically benign but it can evolve to a frank lymphoma in about $8-10 \%$ of cases $[2,9]$.

Type II MC is the final immunochemical pattern of type III and oligoclonal MC. The oligoclonal type appears to be an interme- 
diate stage in the course of type III, changing to type II MC and predisposing HCV-infected patients to develop symptomatic cryoglobulinemic vasculitis [10].

Four IgG subclasses are described: IgG1 (60-65\% of total IgG), IgG2 (20-25\%), IgG3 (5-10\%) and IgG4 (3-6\%) with different chemical and physical properties, whose clinical significance is not fully understood. IgG3 has a 7 day half-life, other IgG subclasses have a longer half-life (about 20 days) [11]. IgG3 are very effective in the induction of effector functions. Being a potent pro-inflammatory antibody, IgG3 shorter half-life may function to limit an abnormal inflammatory response [12].

Analysis of immunoglobulin components in murine CGs has shown selective enrichment of a particular IgG subclass; IgG3. IgG3 monoclonal CGs with RF activity induce extensive extrahepatic manifestations. The human and murine IgG3 have an equal tendency to self-assemble, although there are structural and functional differences, demonstrating a role in the molecular mechanism of the formation of cryoprecipitate $[13,14]$. Human IgG3 myeloma proteins undergo concentration and temperature dependent aggregation, although not always cryoprecipitation [15]. Since the aggregating site seems to be localized in the IgG3 heavy chain Fc fragment, the molecular mechanism of self-association may not be identical in human and murine IgG3. Nevertheless, the physicochemical properties of human IgG3 would explain the frequent enrichment of the human IgG3 subclass in association with IgM RF in MC [13].

This study aims to investigate the presence, prevalence and characteristics of the subclasses of CGs in patients infected with $\mathrm{HCV}$, through the study of various immunochemical patterns in the presence of mono-, oligo- or polyclonal IgG3, to search for a relationship between their occurrence in $\mathrm{HCV}$-positive patients and $\mathrm{MC}$ evolution. We also investigated whether there is an association between the presence of Ig classes and subclasses in cryoprecipitate and RF activation, since this may be useful to better comprehend the molecular mechanisms of mixed cryoglobulinemic vasculitis.

\section{Patients and methods}

\subsection{Patients}

Fifty HCV-infected patients were retrospectively enrolled at the Fondazione Policlinico “A. Gemelli”, Rome, Italy. The inclusion criteria were detectable HCV-RNA in the serum, and the presence of positive cryocrit (CRT); exclusion criteria were a current or a previous antiviral treatment and HIV or HBV co-infection. All patients gave their informed consent, in accordance with the Principles of the Declaration of Helsinki.

\subsection{Clinical and laboratory assessments}

Since CGs detection should be carefully carried out starting from the pre-analytical phase, blood samples were collected and stored according to an accurate procedure [16]. Pre-warmed equipment, rigorously kept at $37^{\circ} \mathrm{C}$, was used for blood collection $(20 \mathrm{~mL})$ in anti-coagulant free tubes. EDTA-containing tubes were used for viremia assessment and microbiology tests.

HCV-RNA detection was performed in both supernatant and cryoprecipitate and was determined through real-time polymerase chain reaction, transcription-mediated amplification, and multiprobe reverse hybridization of the $5^{\prime}$ untranslated region of the HCV genome. The HCV genotype (GT) was determined for each sample by using the line probe assay Versant HCV genotype LiPA 2.0 (Siemens Healthcare Diagnostics, Munich, Germany). Liver disease stage was assessed by liver elastography using FibroScan (Echosens, Paris, France).
The following serum parameters were assessed: IgG, IgA, IgM, C3, C4 (determined at $37^{\circ} \mathrm{C}$, following cryoglobulin separation) and IgG, IgA, IgM supernatant by Nephelometric measurement with Dade Behring BNII (Siemens Healthineers Diagnostics Products $\mathrm{GmbH}$, Marburg, Germany). RF was tested in duplicate at $37^{\circ} \mathrm{C}$, using ELISA kits for IgG-RF (INOVA diagnostics, USA) and nephelometric assays for IgM-RF IU/mL (Siemens Healthineers Diagnostics, Germany); Alanine aminotransferase (ALT) was measured using Advia Chemistry XP (Siemens Healthineers, Germany). All specimens were analyzed at the same time following the manufacturer's instructions.

\subsubsection{Cryoglobulin detection and typing}

CRT was measured for all samples. Samples were allowed to clot for $\geq 1 \mathrm{~h}$ (until complete clotting) prior to centrifugation at $37^{\circ} \mathrm{C}$ at $1700 \times \mathrm{g}$ for $5 \mathrm{~min}$. Separated serum was transferred into Wintrobe tubes, immediately incubated at $4{ }^{\circ} \mathrm{C}$ for 7 days to allow cryoglobulin precipitation and subsequently centrifuged for $10 \mathrm{~min}$ at $1700 \times g\left(\right.$ at $\left.4{ }^{\circ} \mathrm{C}\right)$ [17]. Cryoprecipitate was reported as a percentage of the total serum volume.

The supernatant was then removed and stored for further analyses; the remaining cryoprecipitate was washed 3 times using $2 \mathrm{~mL}$ of a $4 \%$ PEG solution, obtained by mixing $4 \mathrm{~g}$ of polyethylene glycol (PEG 6000) with $100 \mathrm{~mL}$ of phosphate-buffered saline (PBS $4 \%$ $\mathrm{p} / \mathrm{v}$ ). After each wash, the cryoprecipitate sample was centrifuged at $1700 \times g$ for $5 \mathrm{~min}$ at $37^{\circ} \mathrm{C}$ [16]. Cryoprecipitates were resuspended in an appropriate volume of $3 \%$ PEG 6000 solution, and resolubilized for $30 \mathrm{~min}$ at $37^{\circ} \mathrm{C}$.

Immunoglobulins were typed and characterized by immunofixation electrophoresis, optimized for the Easyfix G26 fullyautomated system (Interlab, Italy), according to the manufacturer's instructions. Immunofixation electrophoresis was performed using antisera against $\gamma, \alpha, \mu, \kappa$, and $\lambda$ (supplied by Interlab, Italy) [18].

Cryoprecipitate IgG subclass research was carried out by immunofixation electrophoresis by using antisera against IgG1, IgG2, IgG3 and IgG4 (supplied by Binding Site, England). The purity of cryoprecipitates was checked by running the samples by immunofixation electrophoresis with total protein antiserum.

\subsection{Statistical analysis}

Collected data sets were analyzed using Statgraphics Plus 5.1 (Statpoint Technologies, Inc., Warrenton, VA, USA) and GraphPad (S. Diego, CA, USA). The categorical and quantitative variables are expressed as number, percent and mean \pm standard deviation, respectively. The $\mathrm{X}^{2}$ and Fisher tests were used to evaluate categorical variables. Statistical significance was set at $p<0.05$.

\section{Results}

Fifty HCV-infected patients with circulating CGs (32 women, mean age $58.4 \pm 10.9$ years) were enrolled at the Fondazione Policlinico “A. Gemelli”, Rome, Italy, between January 2013 and January 2015. Patients were stratified into two subgroups according to IgG3 subclass positive or negative; the main demographic, clinical and virological characteristics of the population study are summarized in Table 1.

Significant differences were observed upon comparison of IgM supernatant levels in IgG3-positive and IgG3-negative patients (Fig. 1, panel a), with higher IgM concentration in the IgG3-negative cohort $(p=0.03)$. Likewise, higher IgM-RF concentrations were detected in the IgG3-negative group ( $p=0.01)$ (Fig. 1, panel b). On the other hand, the IgG3-positive group showed higher IgG-RF concentrations compared to the IgG3-negative group $(p<0.0001)$ (Fig. 1, panel c). Among the IgG3-positive patients, the IgG-RF was lower in the IgM-positive group $(p=0.03)$ (Fig. 1, panel d). Six of 
a)

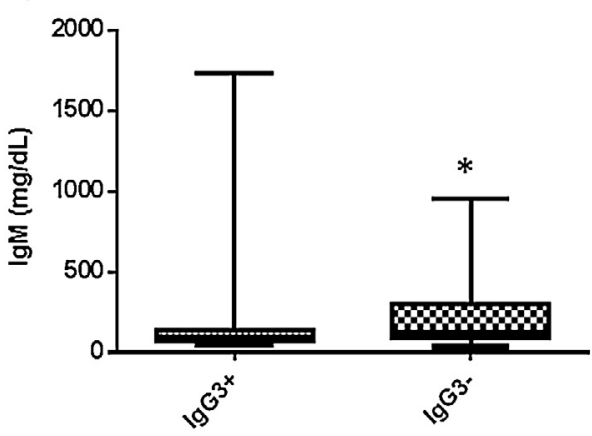

c)

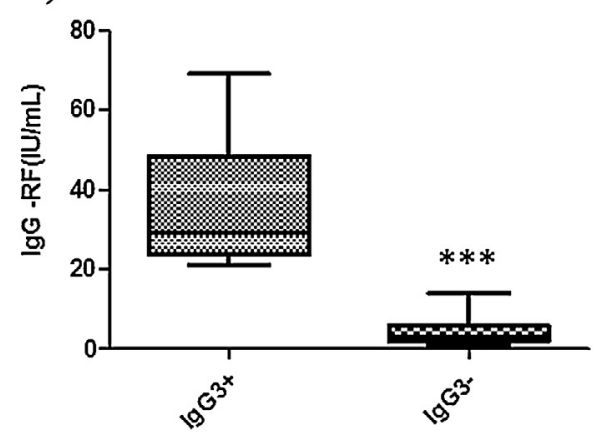

b)

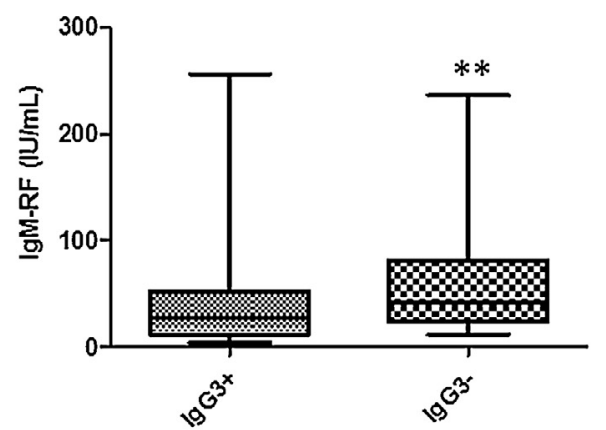

d)

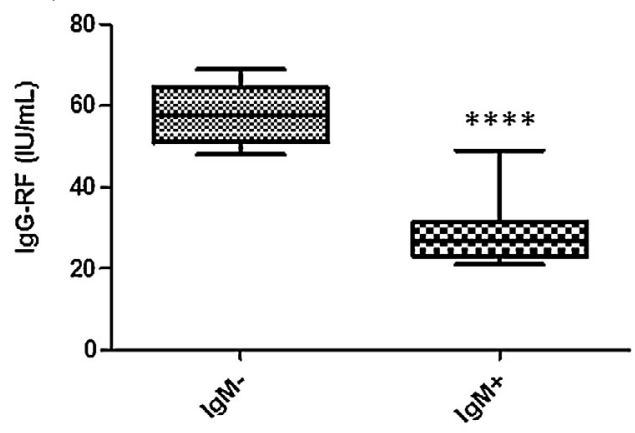

Fig. 1. Results of IgG3-positive vs IgG3-negative and IgM-negative vs IgM-positive in the IgG3-positive group.

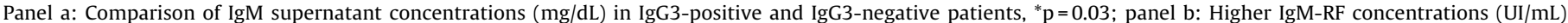

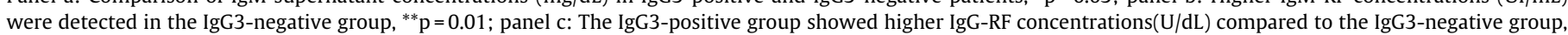
${ }^{* * *} \mathrm{p}<0.0001$; panel d: Among the IgG3-positive patients, the IgG-RF (UI/L) was lower in the IgM-positive group, ${ }^{* * * *} \mathrm{p}=0.03$.

a)

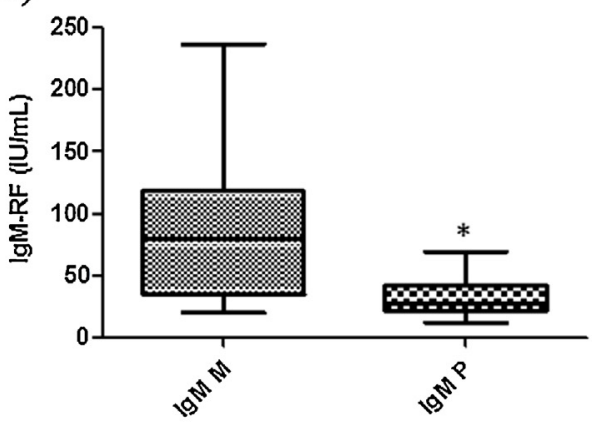

c)

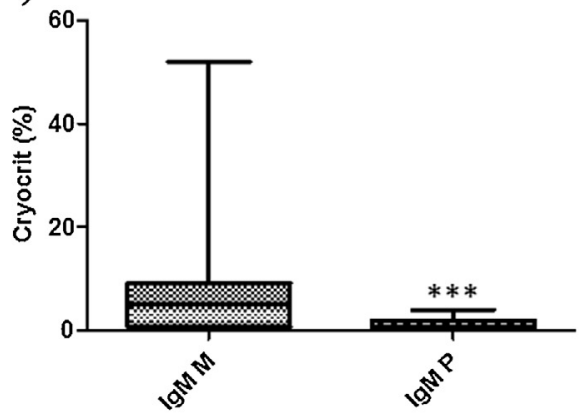

b)

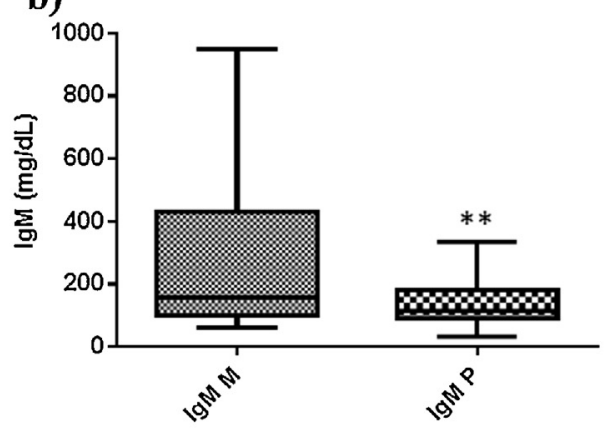

d)

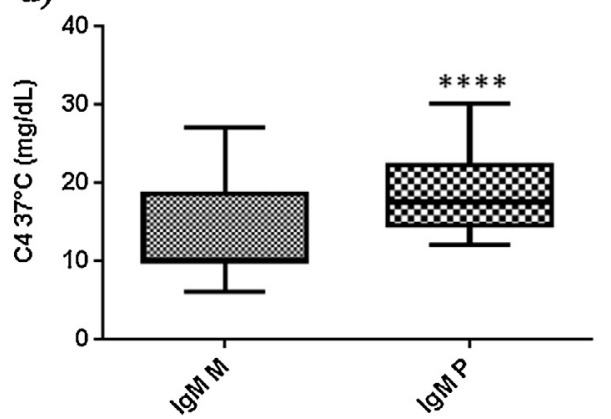

Fig. 2. Results of monoclonal IgM vs polyclonal IgM in the IgG3-negative group.

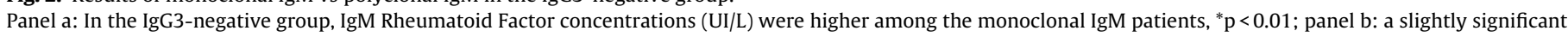

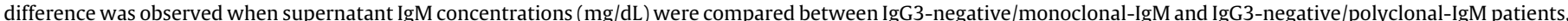

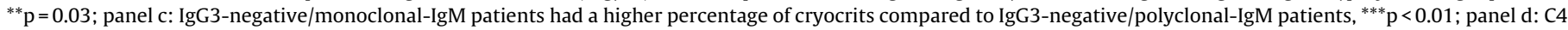
levels $(\mathrm{mg} / \mathrm{dL})$ in the IgG3-negative group were higher in the polyclonal-IgM group as opposed to the polyclonal-IgM cohort, ${ }^{* * * *} \mathrm{p}<0.01$. 
Table 1

Main demographic, hepato-virological and clinical characteristics of the 50 patients.

\begin{tabular}{|c|c|c|c|c|}
\hline & All patients $(n=50)$ & Patients IgG3 positive $(n=26)$ & Patients IgG3 negative $(\mathrm{n}=24)$ & $p$-Value \\
\hline Mean age (years) & $52.18 \pm 12.56$ & $60.6 \pm 10.2$ & $55.9 \pm 11.2$ & \\
\hline \multicolumn{5}{|l|}{ Sex } \\
\hline Male & 18 & 9 & 9 & \\
\hline Female & 32 & 17 & 15 & \\
\hline Histology (\%) & $26^{*}$ & 10 & $16^{*}$ & \\
\hline Chronic hepatitis & 20 & 8 & 12 & \\
\hline Cirrhosis & 4 & 2 & 2 & \\
\hline $\operatorname{ALT}(\mathrm{IU} / \mathrm{mL})$ & $70.7 \pm 70.3$ & $71.3 \pm 85.4$ & $70.1 \pm 51.9$ & NS \\
\hline \multicolumn{5}{|l|}{ METAVIR score (\%) } \\
\hline F0 & 18 & 18 & 0 & \\
\hline $\mathrm{F} 1$ & 28 & 16 & 12 & \\
\hline $\mathrm{F} 2$ & 36 & 12 & 24 & \\
\hline F3 & 18 & 6 & 12 & \\
\hline $\mathrm{C} 3(\mathrm{mg} / \mathrm{dL})$ & $99.7 \pm 59.2$ & $81.1 \pm 41.6$ & $110 \pm 68.2$ & \\
\hline $\mathrm{C} 4(\mathrm{mg} / \mathrm{dL})$ & $17.6 \pm 10.4$ & $19.4 \pm 10.6$ & $16 \pm 9.2$ & \\
\hline Cryocrit (\%) & 3.96 & 3.15 & 4.88 & \\
\hline IgM positive & 44 & 20 & 24 & 0.03 \\
\hline Polyclonal (Type III CG) & 17 & 5 & 12 & \\
\hline Monoclonal (Type II CG) & 27 & 15 & 12 & \\
\hline $\operatorname{IgM~RF}(\mathrm{IU} / \mathrm{mL})$ & $42.4 \pm 42.6$ & $39.8 \pm 51.2$ & $45.5 \pm 31.7$ & 0.01 \\
\hline $\operatorname{IgG~RF~(IU/mL)~}$ & $20.5 \pm 18.9$ & $22 \pm 20.4$ & $18.8 \pm 17.1$ & $<0.0001$ \\
\hline $\operatorname{IgG1}(\%)$ & 100 & 50 & 50 & \\
\hline $\operatorname{IgG2}(\%)$ & 20 & 12 & 8 & \\
\hline $\operatorname{IgG} 4(\%)$ & 4 & 4 & 0 & \\
\hline Viral titer supernatant $(\mathrm{IU} / \mathrm{mL} \times 106)$ & 2.3 & 2.13 & 2.55 & \\
\hline Viral titer cryoprecitate $(\mathrm{IU} / \mathrm{mL} \times 106)$ & 3.82 & 3.58 & 4.08 & \\
\hline HCV genotype & $1 \mathrm{a}(13) ; 1 \mathrm{~b}(24) ; 2 \mathrm{a}(9) ; 2 \mathrm{a}-3 \mathrm{c}(3)$ & $1 \mathrm{a}(8) ; 1 \mathrm{~b}(10) ; 2 \mathrm{a}(4) ; 2 \mathrm{a}-3 \mathrm{c}(3)$ & $1 \mathrm{a}(5) ; 1 \mathrm{~b}(14) ; 2 \mathrm{a}(5)$ & \\
\hline
\end{tabular}

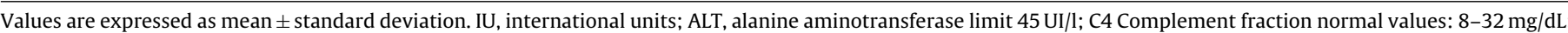
* $\mathrm{p}=0.03$; difference of IgM supernatant concentrations ( $\mathrm{mg} / \mathrm{dL})$ between IgG3-negative/monoclonal-IgM and IgG3-negative/polyclonal-IgM patients.

the IgG3 patients with RF activities were also IgG1 positive. We then analyzed the IgG3-negative group in more detail and found that IgM-RF levels were higher among the monoclonal IgM patients compared to polyclonal IgM patients $(p<0.01)$ (Fig. 2, panel a). We also observed a difference in total IgM concentrations between the same groups ( $p=0.03$ ) (Fig. 2, panel b).

IgG3-negative/monoclonal IgM patients also had higher CRT concentrations compared to IgG3-negative/polyclonal IgM patients $(p<0.01)$ (Fig. 2, panel c), whereas no differences were observed among the IgG3-positive group (data not shown). At the same time, C4 levels were higher in the polyclonal IgM group compared to the monoclonal IgM group $(p<0.01)$ (Fig. 2, panel $d$ ), whereas no differences were observed in C3 levels.

Among IgG3-positive CGs group, 9 patients (34.5\%) had a METAVIR score $=$ F0, 8 patients $(31 \%)$ had $F 1,6$ patients $(23 \%)$ had F2 and 3 patients (11.5\%) had F3; no CGs patients with negative IgG3 showed a F0 METAVIR score, while $6(25 \%)$ patients had F1, 12 patients (50\%) had F2 and $6(25 \%)$ patients had F3 (Fig. $3 \mathrm{~A}$ ). Considering the monoclonal IgM group, 3 patients (11\%) had F0, 7 (26\%) patients had F1, 8 patients (30\%) had F2 and the remaining 9 patients (33\%) had F3; there were no patients in the polyclonal IgM group with an F0 and F3 METAVIR score while 7 patients (41\%) had F1 and 10 patients (59\%) were F2 (Fig. 3B).

\section{Discussion}

Over $90 \%$ of Type II and Type III MC cases are HCV-induced, although other viruses have also been related to this condition $[19,20]$.

CGs represent only a part of the circulating immune complexes and their cryoprecipitable fraction can vary widely from person to person and in the same individual during the course of the disease [21].

The presence of hyperstimulation of the immune system by infectious agents such as viruses and the chronic inflammatory response can lead to the development of autoimmune diseases with loss of control of B and T cells [22]. A possible molecular mimicry of
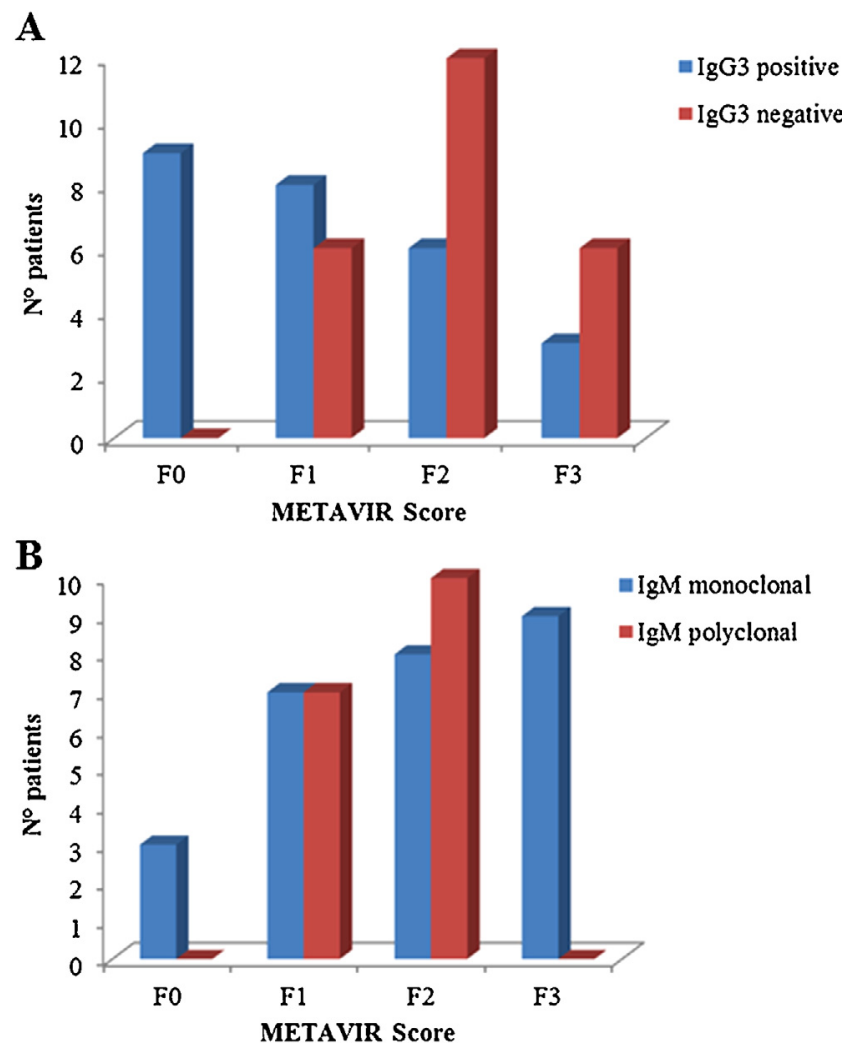

Fig. 3. METAVIR score of Cryoglobulin patients.

Panel A: IgG3-positive group: 34.5\% METAVIR score =F0, 31\% F1, 23\% F2 and 11.5\% F3; IgG3-negative group: 0\% METAVIR score $=\mathrm{F0}, 25 \% \mathrm{~F} 1,50 \% \mathrm{~F} 2$ and $25 \% \mathrm{~F} 3$.

Panel B: IgM monoclonal group: 11\% METAVIR score = F0, 26\% F1, 30\% F2 and 33\% F3;

IgM polyclonal group: 0\% METAVIR score = F0 and F3, 41\% F1 and 59\% F2. 


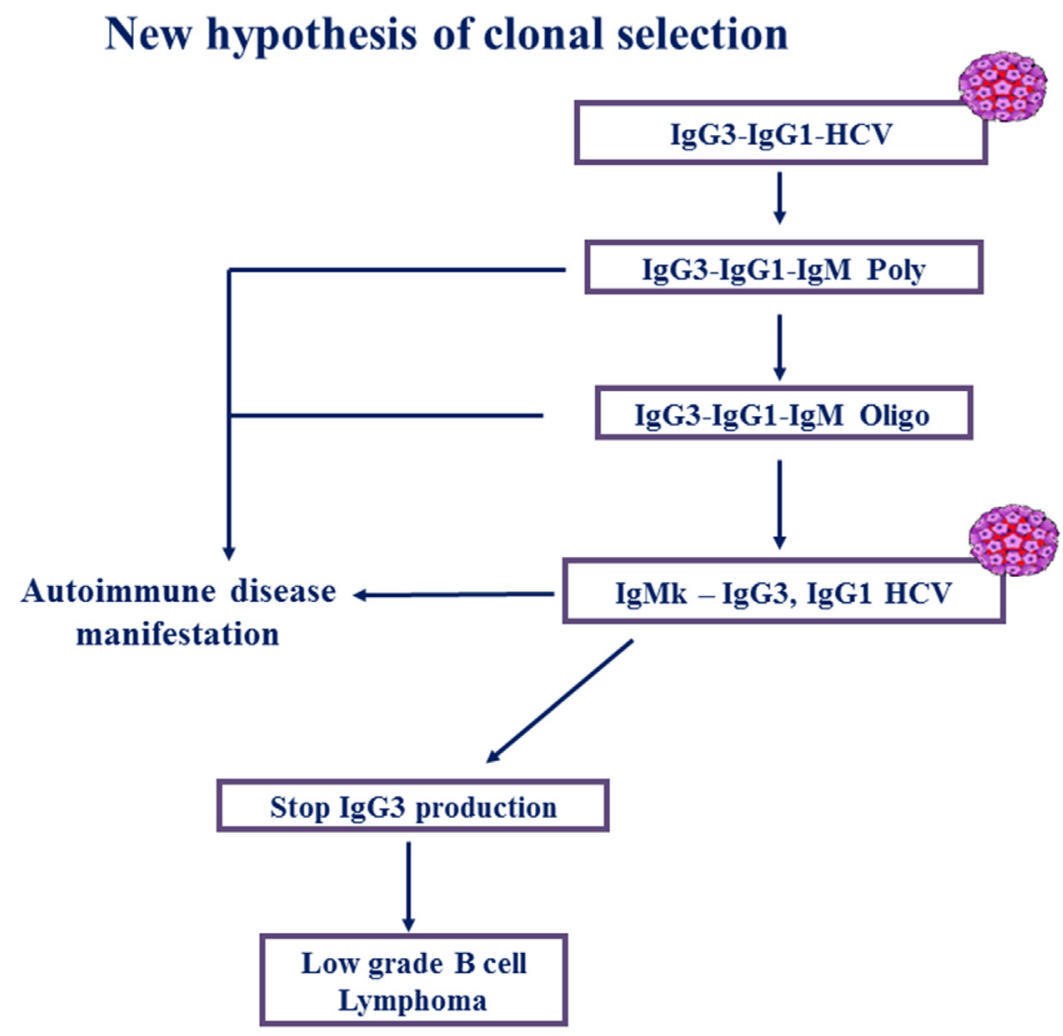

Fig. 4. New hypothesis of clonal selection.

HCV could be superimposed on the natural versus host due to the chronicity of the disease [23].

In a previous study on naive HCV patients, anti-nuclear antibodies were found in subjects without extrahepatic disease [24]. Serum IgG subclass distribution in patients with autoimmune diseases is different from that found in healthy people. Zhang et al. showed that IgG3 subclass levels in serum present distinct patterns in different autoimmune diseases. In primary biliary cirrhosis IgG3 levels are prominently increased compared with those in the other autoimmune diseases showing how this subclass is also involved in autoimmune liver diseases [25].

The expansion of $\mathrm{B}$ cells contributes to autoantibody production, such as anti-IgG RF that constitutes the IgG-IgM immune complexes, commonly found in CG precipitates [21]. In this study, higher concentrations of IgM-RF were detected in the supernatant of the IgG3-negative samples compared to IgG3-positive ones. The expansion of the IgM-RF clone could be due to the persistence of antigenic stimulus; on the other hand, the higher levels of IgG-RF in the IgG3-positive group compared to the negative one may indicate a more recent contagion. Being that $\mathrm{HCV}$ infection is a progressive disease we can use the METAVIR score as a surrogate indicator of the duration of infection. In fact patients with IgG3 presented a METAVIR score between F0 and F1(Fig. 3A), while IgG3 negative patients with monoclonal IgM, had a higher METAVIR score (Fig. 3B).

We also showed that, in the IgG3-negative group, the levels of IgM-RF are higher in patients with monoclonal IgM than in patients with polyclonal IgM. The presence of monoclonal IgM is associated with higher CRT and lower C4 levels, leading us to speculate that patients with monoclonal IgM show a higher activation of the cryoglobulinemic clone.

Thus, the presence of IgG3 in cryoprecipitates suggests that the immune system is highly stimulated [26] and thus more susceptible to the development of autoimmune mechanisms. These markers may be a prognostic factor for the onset of autoimmune disease in
HCV-infected individuals. IgG3 is particularly effective in the induction of effector functions. It is a potent pro-inflammatory antibody, its shorter half-life may limit a potential, abnormal inflammatory response. Viral infections usually lead to the onset of IgG1 and IgG3 subclasses, with IgG3 antibodies appearing first in the course of the infection. IgG3-dominated responses appear to be rare [27].

The sporadic presence of other IgG subclasses (IgG2, IgG4) in cryoprecipitate could be due to a possible non-specific entrapment during the early formation of IgG1-IgG3 RF and IgG1-IgG3-IgM RF immune complexes.

The three serological types of MC, Type III, Type II-III or Type II MC, may represent different steps of an evolving autoimmune disorder, beginning with polyclonal B-cell activation and leading to the final oligo-monoclonal lymphoproliferative stage, (Type II MC) $[20,21]$. In fact, CGs could be part of a progressive clonal selection process, in which B-cells are initially stimulated to produce more clones of oligoclonal immunoglobulin IgG3 with RF activity. Foregoing results indicated that the presence of $\operatorname{IgG} 3$ in cryoprecipitates of HCV and antinuclear antibody-positive patients are probably the main factor activating autoimmune mechanisms in the long term (Fig. 4) [7]. Interestingly, the association between the CRT level and the immunochemical pattern seems to confirm the hypothesis of clonal selection: lower CRT levels were found in both polyclonal and oligoclonal Type III CGs, while the highest were detected in Type II [26].

The simultaneous presence of IgG-RF with an immunochemical pattern in which we observed the presence of IgG3, supports the hypothesis of an involvement of this subclass in the initiation of early stages of CGs [7]. It can therefore be assumed that the CGs were originally formed only by two classes of IgG: IgG1 and IgG3 with RF activity, and that their presence might be interpreted as an early stage MC early stage. In the initial stage at the beginning of HCV infection, the IgG1 recognizes the virus (Fig. 5, panel A) and forms a complex that activates the IgG3 RF, causing the precipitation of the circulating immune complexes (Fig. 5, panel B). 

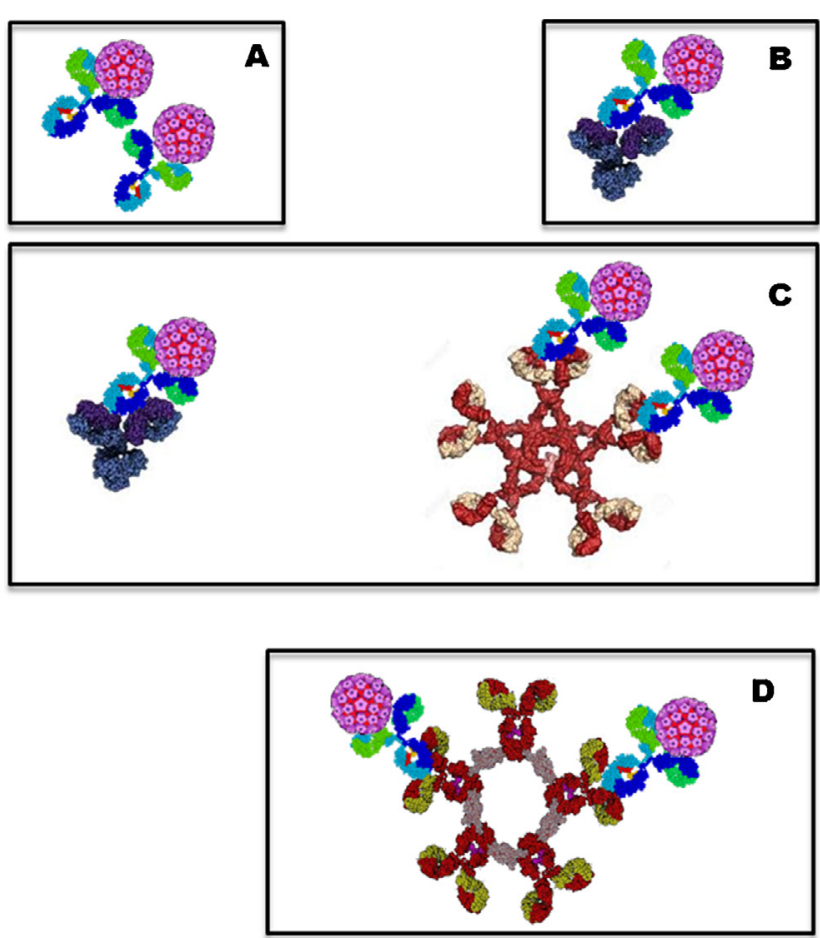

$\lg G 1$

$\lg \mathbf{3} 3$

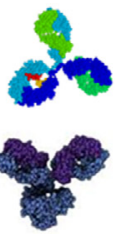

c

$\lg M$

Polyclonal

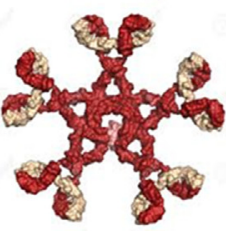

$\lg M$

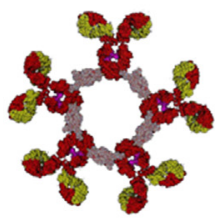

$\mathrm{HCV}$

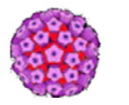

Fig. 5. Cryoglobulinemic staging.

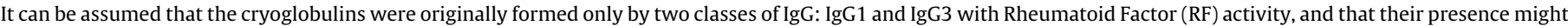

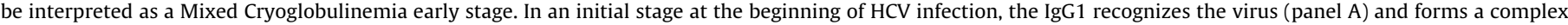

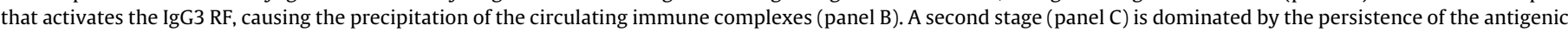

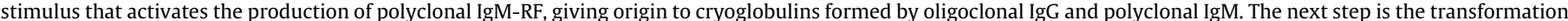
of the polyclonal IgM-RF to oligoclonal through a clonal selection system with the simultaneous presence of an IgG3 RF characterized by oligoclonal cryoglobulins.

The last stage (panel D) involves the formation of a monoclonal IgM-RF clone that can be present, for a period of time, together with a monoclonal IgG3 RF clone.

A second stage (Fig. 5, panel C) is dominated by the persistence of antigenic stimulus that activates the production of polyclonal IgM-RF, giving origin to CGs formed by oligoclonal IgG and polyclonal IgM. The next step is the transformation of the polyclonal IgM-RF to oligoclonal through a clonal selection system with the simultaneous presence of an IgG3 RF characterized by oligoclonal CGs.

The last stage (Fig. 5, panel D) involves the formation of a monoclonal IgM-RF clone that can be present, for a period of time, together with a monoclonal IgG3 RF clone.

The results of our study help to clarify the molecular basis of the CGs generation in HCV-related MC. The presence of small concentrations of cryoprecipitate and its immunochemical characterization could be useful in the clinical management of these patients, providing important information about the stage of the disease.

A more accurate method of detecting CGs could increase the prevalence in HCV patients who are positive for these proteins and in association with related symptoms, make them eligible for antiviral treatment even in the absence of severe liver disease.

Furthermore, the detection of increased levels of IgG-RF and the presence of IgG3 could suggest that scrupulous follow-ups should be carried out for these patients to identify the evolution towards an autoimmune or lymphoproliferative disease in the early phase.

In conclusion, our study proposes that IgG3 subclasses can be used to predict the evolution of CGs transformations, responsible for extrahepatic manifestations in more advanced stages.

\section{Conflict of interest}

None declared.

\section{Guarantor}

UB.

\section{Author contributions}

All the authors have accepted responsibility for the entire content of this submitted manuscript and approved submission.

\section{Acknowledgements}

The authors would like to thank Helena Ritchie and Mary Forrest for the English language editing of the manuscript.

The work of the Center for Systemic Manifestations of Hepatitis Viruses (MaSVE) of the University of Florence was supported by grants from the "Associazione Italiana per la Ricerca sul Cancro” (AIRC), IG 2015 Id.17391,“Istituto Toscano Tumori” (ITT), “Ente Cassa di Risparmio di Firenze" and Fondazione "Oretta Bartolomei Corsi".

\section{References}

1] Mohd Hanafiah K, Groeger J, Flaxman AD, et al. Global epidemiology of hepatitis $C$ virus infection: new estimates of age-specific antibody to HCV seroprevalence. Hepatology 2013;57:1333-42.

2] Zignego AL, Gragnani L, Giannini C, et al. The hepatitis C virus infection as a systemic disease. Intern Emerg Med 2012:7(Suppl. 3):S201-8.

[3] Bachy E, Besson C, Suarez F, et al. Hepatitis C virus infection and lymphoma. Mediterr J Hematol Infect Dis 2010;2.

[4] Ferri C, Pileri S, Zignego AL. Hepatitis C virus, B-cell disorders, and nonHodgkin's Lymphoma. Infect Causes Cancer 2000:349-68.

[5] Wintrobe MM, Buell MV. Hyperproteinemia associated with multiple myeloma with report of a case in which an extraordinary hyperproteinemia was associ- 
ated with thrombosis on the retinal veins and symptoms suggesting Raynaud's disease. Bull Johns Hopkins Hosp 1933;52:156-65.

[6] Ferri C, Zignego AL, Pileri A. CGs. J Clin Pathol 2002;55:4-13.

[7] Basile U, Gulli F, Torti E, et al. Anti-nuclear antibody detection in cryoprecipitates: distinctive patterns in hepatitis C virus-infected patients. Dig Liv Dis 2015;47:50-6.

[8] Misiani R, Bellavita P, Fenili D, et al. Hepatitis C virus infection in patients with essential mixed cryoglobulinemia. Ann Intern Med 1992;117:573-7.

[9] Vallat L, Benhamou Y, Gutierrez M, et al. Clonal B cell populations in the blood and liver of patients with chronic hepatitis $C$ virus infection. Arthritis Rheum 2004:50:3668-78.

[10] Gulli F, Santini SA, Napodano C, et al. Cryoglobulin test and cryoglobulinemia hepatitis C-virus related. Mediterr J Hematol Infect Dis 2017;9:e2017007.

[11] Puissant-Lubrano $B$, Peres $M$, Apoil PA, et al. Immunoglobulin IgA, IgD, IgG, IgM and IgG subclass reference values in adults. Clin Chem Lab Med 2015;53:e359-61.

[12] Vidarsson G, Dekkers G, Rispens T. IgG subclasses and allotypes: from structure to effector functions. Front Immunol 2014:5:520.

[13] Izui S, Bemey T, Shibata T. IgG3 CGs in autoimmune MRL-lpr/lpr mice: immunopathogenesis, therapeutic approaches and relevance to similar human diseases. Ann Rheum Dis 1993;52:S48-54.

[14] Otani M, Kuroki A, Kikuchi S, et al. Sialylation determines the nephritogenicity of IgG3 CGs. J Am Soc Nephrol 2012;23:1869-78.

[15] Nishimura Y, Nakamura H. Human monoclonal cryoimmunoglobulins. I. Molecular properties of IgG3 kappa (Jir protein) and the cryo-coprecipitability of its molecular fragments by papain. J Biochem 1984;95:255-65.

[16] Basile U, Torti E, Dell'Abate MT, et al. Pre-analytical phase in cryoglobulin (CRG) detection: an alternative method for sample transport. Clin Chem Lab Med $2015 ; 20$.
[17] Sargur R, White P, Wegner W. Cryoglobulin evaluation: best practice. Ann Clin Biochem 2010;47:8-16.

[18] Alper CA. Johnson AM immunofixation electrophoresis: a technique for the study of protein polymorphisms. Vox Sangui 1969;17:445-52.

[19] Retamozo S, Brito-Zerón P, Bosch X, et al. Cryoglobulinemic disease. Oncology 2013;27:1098-105.

[20] Zignego AL, Gragnani L, Piluso A, et al. Virus-driven autoimmunity and lymphoproliferation: the example of HCV infection. Expert Rev Clin Immunol 2015;11:15-31.

[21] Ferri C, Sebastiani M, Giuggioli D, et al. Hepatitis C virus syndrome: a constellation of organ- and non-organ specific autoimmune disorders, B-cell non-Hodgkin's lymphoma, and cancer. World J Hepatol 2015;7:327-43.

[22] Shoenfeld Y. Everything is autoimmune until proven otherwise. Clinic Rev Allerg Immunol 2013;45:149-51.

[23] Cacoub P, Gragnani L, Comarmond C, et al. Extrahepatic manifestations of chronic hepatitis C virus infection. Dig Liver Dis 2014;15(Suppl. 5):S165-73.

[24] Gulli F, Basile U, Gragnani L, et al. Autoimmunity and lymphoproliferation markers in naïve HCV-RNA positive patients without clinical evidences of autoimmune/lymphoproliferative disorders. Dig Liver Dis 2016:24.

[25] Zhang H, Li P, Wu D, et al. Serum IgG subclasses in autoimmune diseases. Med (Baltimore) 2015;94:e387.

[26] Bellotti V, Zorzoli I, Bossi A, et al. Immunochemical characteristics of a particular cryoglobulin. A new cryoglobulin subgroup? Clin Exp Rheumatol 1991;9:399-402.

[27] Ferrante A, Beard LJ, Feldman RG. IgG subclass distribution of antibodies to bacterial and viral antigens. Pediatr Infect Dis J 1990;9(Suppl. 8):16-24. 